\title{
Agarose Gel Analysis of 15-40-kb PCR Amplimers
}

\author{
Valerie Burland, Foner P. Curtis and Noriko Kusukawa \\ FMC BioProducts, Rockland, ME, USA
}

BioTechniques 21:142-144 (July 1996)

\begin{abstract}
Accurate resolution of PCR products in the range of $15-40 \mathrm{~kb}$ may be obtained in agarose gels without pulsed-field electrophoresis. A gel of $0.3 \%$ SeaKem ${ }^{\circledR}$ Gold agarose cast on GelBond ${ }^{\circledR}$ support film provides good resolution and sufficient gel strength to reliably allow staining and photography. This paper describes a test system for Long PCR and demonstrates analysis of the PCR products on a gel run under standard low-voltage electrophoresis conditions.
\end{abstract}

\section{INTRODUCTION}

Amplification of target fragments in the kilobase range by Long polymerase chain reaction (Long PCR) has become possible by using a combination of thermostable polymerases with and without the proofreading activity of 3 ' exonuclease (1-3). The addition of a proofreading enzyme in very small amounts allows removal of mismatched nucleotides without degradation of oligonucleotide primers (1), generating longer extension products at high fidelity $(1,4)$. For analysis of PCR products, standard agarose gel procedures resolve fragments well up to about $20 \mathrm{~kb}$, but it has been necessary to use pulsed-field electrophoresis for accurate analysis of fragments between 20 and $40 \mathrm{~kb}$. To simplify this process, we screened for a high gel strength agarose, which could be used under standard electrophoresis conditions (without pulsedfield) to obtain good resolution of these large DNA fragments. SeaKem ${ }^{\circledR}$ Gold (FMC BioProducts, Rockland, ME, USA), with the highest gel strength of all the available agaroses, met these requirements at a concentration of $0.3 \%$. Although other agaroses theoretically have the resolving power to separate fragments in this range (e.g., SeaKem LE and FastLane ${ }^{\circledR}$ agaroses, both from FMC BioProducts), they are not practical alternatives, since at the low concentrations required, their gel strengths are too low to allow the routine handling of staining and photography.

\section{DISCUSSION}

The LA PCR kits Versions 1 and 2 from Takara (PanVera, Madison, WI, USA) were used to generate a series of amplified fragments from $15-40 \mathrm{~kb}$ with very little modification of the suggested protocols. The template used was purified phage lambda DNA. A set of primers was prepared consisting of one forward primer and a series of reverse primers 31-32 bases long, whose target sites in the template were spaced at 5 -kb intervals to give amplimers from $15-40 \mathrm{~kb}$. The primer sequences were from the product insert in the Expand ${ }^{\mathrm{TM}}$ Long template PCR system (Boehringer Mannheim, Indianapolis, IN, USA). They were synthesized at Genosys (The Woodlands, TX, USA) with cartridge purification.

Reaction mixtures were set up as specified in the Takara product literature and using AmpliWax ${ }^{\mathrm{TM}}$ PCR Gems (PerkinElmer, Norwalk, CT, USA) to facilitate the hot-start procedure. The Version 1 PCR kit amplified the 15-30-kb fragments well. The Version 2 kit readily produced good yields of 35- and 40-kb targets when the magnesium concentration was increased to 5.0 and $5.4 \mathrm{mM}$, respectively, with appropriate thermal cycling. Reactions were performed in a PTC-100 ${ }^{\mathrm{TM}}$ Model 60 Thermal Cycler (MJ Research, Watertown, MA, USA). For all reactions, the block was preheated to $98^{\circ} \mathrm{C}$ before putting in the tubes. The 15-30-kb targets were amplified successfully by 30 cycles of a simple two-step cycle: $98^{\circ} \mathrm{C}$ for $20 \mathrm{~s}$, then $68^{\circ} \mathrm{C}$ for $15 \mathrm{~min}$. For the two largest fragments, an extra step was included at the start $\left(94^{\circ} \mathrm{C}\right.$ for $\left.1 \mathrm{~min}\right)$, and a longer extension time (20 min) was used for the first $14 \mathrm{cy}-$ cles. Then for the remaining 16 cycles, the extension time was further increased by $15 \mathrm{~s}$ more in each successive cycle.

The PCR products were analyzed on $0.3 \%$ SeaKem Gold agarose gels in $1 \times$ TAE buffer $(40 \mathrm{mM}$ Tris-acetate, $1.0 \mathrm{mM}$ EDTA, pH 8.3), which gave the optimum combination of resolution and gel strength at low concentration. The results are shown in Figure 1. The gel must be at least $15-\mathrm{cm}$-long for good separation of DNA bands in the $15-40-\mathrm{kb}$ range. For ease of handling, gels of 2.5-mm-thickness were cast either in 


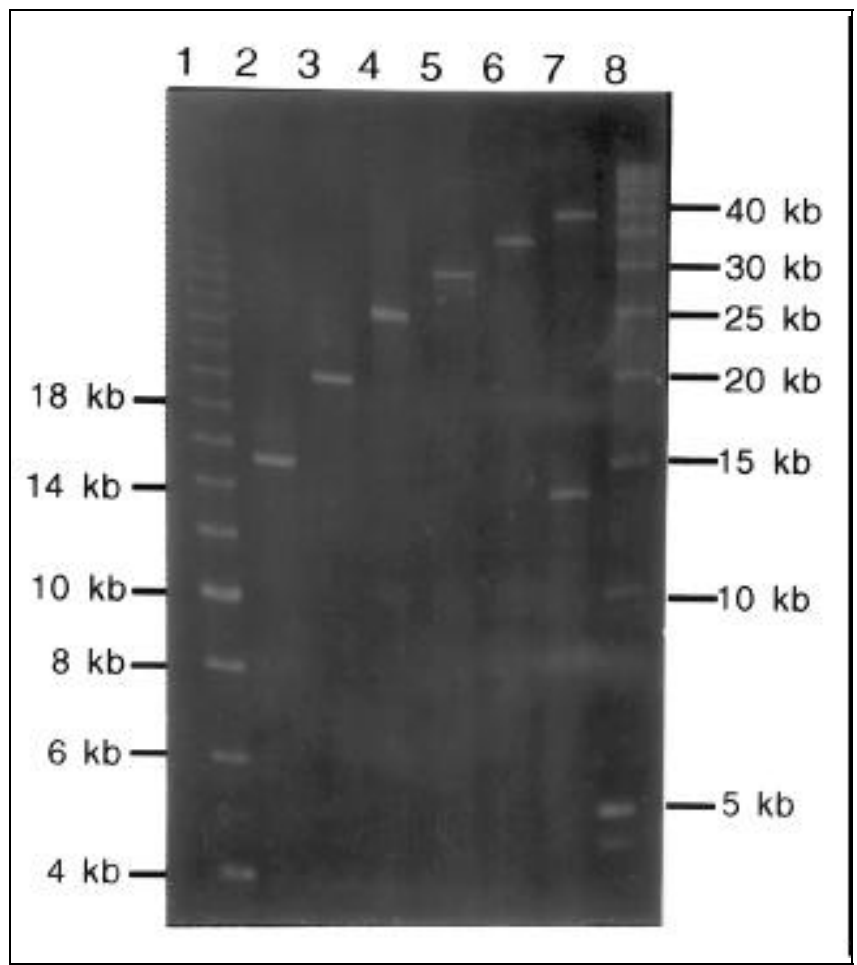

Figure 1. Long PCR products analyzed on $0.3 \%$ SeaKem Gold agarose cast and photographed on GelBond support film. Lane 1, 2-kb ladder (100 ng); lane 8, 5-kb ladder (100 ng). Lanes 2-7, amplified fragments loaded with approximately equal amounts of DNA: $15 \mathrm{~kb}(0.25 \mu \mathrm{L}$ of the unpurified PCR $), 20 \mathrm{~kb}(0.35 \mu \mathrm{L}), 25 \mathrm{~kb}(2.0 \mu \mathrm{L}), 30 \mathrm{~kb}(4.0 \mu \mathrm{L}), 35 \mathrm{~kb}(2.0 \mu \mathrm{L})$ and 40 $\mathrm{kb}(4.0 \mu \mathrm{L})$. The length of the gel portion in the photograph is $10.5 \mathrm{~cm}$, and the $40-\mathrm{kb}$ band has migrated approximately $5.5 \mathrm{~cm}$. Photography with Polaroid Type 55 film. Lane 7 also contains an artifact band at about $14 \mathrm{~kb}$.

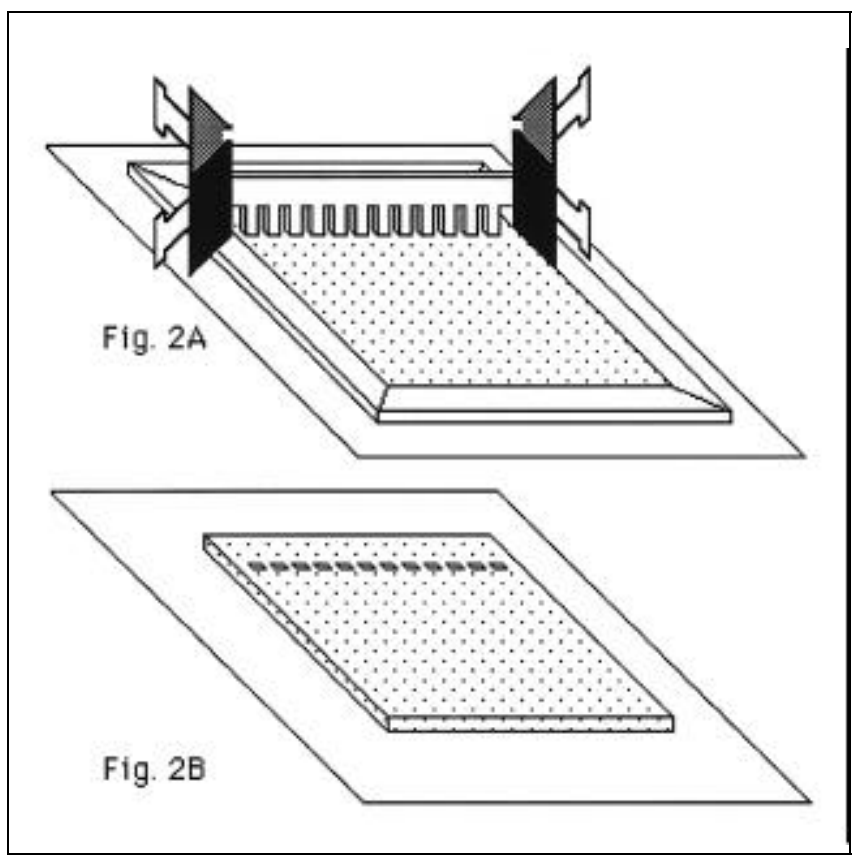

Figure 2. A plastic frame was used to cast $0.3 \%$ agarose gels on GelBond. (A) Strips 1.5 -cm-wide were cut from 3-mm-thick plastic sheet and cemented together to form a rectangle of internal dimensions of $17 \times 9.5 \mathrm{~cm}$. To cast the gel, the frame was placed on GelBond film and weighted. The edge was sealed by pipetting gel solution at about $45^{\circ} \mathrm{C}$ around the inside perimeter. About 1 min later, the bulk of the gel solution was poured in and the comb was placed, supported by spring binder clips. (B) After the gel solidified, the comb and frame were removed, leaving a border of film for handling. 
a frame of $1 \%$ agarose or on GelBond ${ }^{\circledR}$ support film (FMC BioProducts). Unsupported $0.3 \%$ gels are surprizingly resilient and flexible but do take some practice to handle. Gels of $0.2 \%$ SeaKem Gold agarose, while giving excellent separation of the largest fragments, are fragile and difficult to manipulate even with support film.

The gel shown in Figure 1 was cast on GelBond support film using a workshop-made acetal plastic frame to contain the gel and leave a border of film around its edge for handling (Figure 2). To preserve the wells, after the gel was completely set, buffer was layered with a transfer pipet onto the gel surface in the well area as the comb was removed, so that the wells filled immediately. The supported gel was then peeled away from the frame, leaving a border of film for handling. PCR products were loaded along with $2-\mathrm{kb}$ and $5-\mathrm{kb}$ ladders (Superladder High; GenSura Laboratories, Del Mar, CA, USA) as size markers. Electrophoresis was carried out at 1 $\mathrm{V} / \mathrm{cm}$ for $16 \mathrm{~h}$ at room temperature. Shortening the electrophoresis time below $10 \mathrm{~h}$ or increasing the voltage twofold both markedly decreased resolution of fragments above 25 $\mathrm{kb}$. Overloading the DNA also decreased resolution; between 15 and $40 \mathrm{ng}$ should be loaded. The run time is comparable with pulsed-field electrophoresis since a field inversion gel electrophoresis (FIGE) or contour-clamped homogeneous electric field (CHEF) gel for this range of fragments sizes requires 9-12 h, an overnight gel run for most laboratories.

The gel was stained after electrophoresis with ethidium bromide and photographed gel-side down on the transilluminator. GelBond appears fluorescent under UV light since the
UV wavelength is blocked. However, the emission wavelength of ethidium bromide is not blocked; therefore, even though the DNA bands were not easily visible by eye, clear pictures were readily obtained by using Polaroid Type 57 or 55 film (Polaroid, Cambridge, MA, USA) with only small amounts of DNA loaded, at settings of $f$ stop 4.5, exposure time $1 \mathrm{~s}$ for Type 57 and $60 \mathrm{~s}$ for Type 55, using both orange and UV filters for both film types.

\section{ACKNOWLEDGMENT}

We thank Maxine Dumais for helpful advice and discussions concerning agarose.

\section{REFERENCES}

1.Barnes, W.M. 1994. PCR amplification of up to 35-kb DNA with high fidelity and high yield from bacteriophage lambda templates. Proc. Natl. Acad. Sci. USA 91:2216-2220.

2.Cheng, S., C. Fockler, W.M. Barnes and R. Higuchi. 1994. Effective amplification of long targets from cloned inserts and human genomic DNA. Proc. Natl. Acad. Sci. USA 91:5696-5699.

3.Foord, O.S. and E.A. Rose. 1994. Long-distance PCR. PCR Methods Appl. 3:S149-S161.

4.Stewart, A.-C.M., P.E. Gravitt, S. Cheng and C.M. Wheeler. 1995. Generation of entire human Papillomavirus genomes by Long PCR: frequency of errors produced during amplification. Genome Res. 5:79-88.

Address correspondence to Valerie Burland, FMC BioProducts, 191 Thomaston Street, Rockland, ME 04841, USA. Internet: valerie_burland@fmc.com 